\title{
Microscopy and Image Processing Recordkeeping: Never Again Lose Track of Your Metadata
}

\author{
François Robert ${ }^{1}$, Nicolas Piché ${ }^{1}$ and Mike Marsh ${ }^{2}$ \\ ${ }^{1}$ Object Research Systems, Montreal, Quebec, Canada, ${ }^{2}$ Object Research Systems, Denver, Colorado, United \\ States
}

Scientific imaging and subsequent image processing can involve countless parameters. Proper bookkeeping of imaging and image processing operations and associated parameters is tedious and error-prone, but these metadata are highly valuable for image interpretation and reproducibility for experiments in general, and scientific imaging in particular. Core laboratory work benefits when imaging protocols are detailed enough to share and inform other experiments, especially when the documentation suffices to make experiments reproducible. Using the industry-backed open source project, Hyperledger fabric [1], we have developed and describe here the blockchain image management system (BIMS), a open solution that encodes metadata records in a secure ledger that is access-restricted, tamper-proof, fault-tolerant, and collaboration-ready.

The BIMS solution serves the joint purposes of capturing all relevant metadata and then encoding it with utmost security and data integrity so that it will never be lost. The standard for lab notebooks and other recordkeeping should be that enough details are documented for others to reproduce the experiments. This standard is easy to understand for the activities of image acquisition and image processing. For microscopy, every user-accessible parameter on the microscope, from optics to digitization, must be recorded. For image processing, every operation that transforms any of the pixel data must be logged.

We provide a reference implementation for how image processing software can meet the reproducibility standard described above. Dragonfly, commercially-supported image processing software which is free for non-commercial use, includes a number of utilities that range from image enhancement to image segmentation. Like any software, it is important to record not only the operations performed, but the specific parameters. We have extended the Save Image capability of Dragonfly; specifically, we have taken Dragonfly's action log--a detailed recording of every operation performed by the user--and redact all the logged operations that do not affect the image of interest, and then save the relevant details encoded in the saved image file. Because this action log captures pure Python code (in addition to human-readable documentation), the saved operations and parameters establish a reproducibility macro. The software includes a Macro Player that can reproduce this sequence of operations on the same data, or related data, even for distributed batch processing [1].

Blockchain is a technology for recording transactions in a secure and distributed manner. The goals of blockchain are data integrity and data security. No user can contribute nor delete any detail to the ledger without proper authorization. Ledger updates cannot commence, unless distributed copies of the ledger are updated in a synchronized fashion. The result is a digital file that all stakeholders know has not been tampered with, is invulnerable to isolated computer failures, and can be shared across organizations. For this BIMS implementation, we developed with Hyperledger Fabric 1.4, an open-source, high-performance blockchain platform originally contributed by IBM and Digital Asset, and now hosted by the Linux Foundation.

This solution is applied here to imaging and image processing, but it is easily extended to other scientific endeavors. There is great interest in making sure all scientific activity can be reproducible. Because of the openness of this BIMS platform, any scientific software can be extended to document workflows as well as harvest data from the the shared ledgers. 
About macro

\section{1. createDatasetFromFiles}

Creates a dataset from a set of files.

2. publish

Publishes an object.

3. enable interactive threshiolding

Enables or disabled the second leveling of a visual associated to a dataset.

4. setRangeAsUpperOtsu

Sets the range of the second leveling of a visual associated to a dataset to upper Otsu.

5. invert

Reverse the inclusion state of each voxel.

6. overwriteData

Writes a given value in a dataset at the location of the ROI.

7. change image name

- Parameter editor

$\square$ Automatically select the only occurrence when there is a single element available

IDI IID

Figure 1. Dialog showing the MacroPlayer view of recorded operations that document and can reproduce image processing workflow.

\section{References}

B. Provencher, et al., Microscopy and Microanalysis 25 (Suppl 2) (2019), p. 1388-1389. 